KYUNGPOOK Math. J. 53(2013), 117-124

http://dx.doi.org/10.5666/KMJ.2013.53.1.117

\title{
The Polynomial Numerical Index of $L_{p}(\mu)$
}

\section{Sung Guen KIm}

Department of Mathematics, Kyungpook National University, Daegu 702-701, Republic of Korea

e-mail: sgk317@knu.ac.kr

Abstract. We show that for $1<p<\infty, k, m \in \mathbb{N}, n^{(k)}\left(l_{p}\right)=\inf \left\{n^{(k)}\left(l_{p}^{m}\right): m \in \mathbb{N}\right\}$ and that for any positive measure $\mu, n^{(k)}\left(L_{p}(\mu)\right) \geq n^{(k)}\left(l_{p}\right)$. We also prove that for every $Q \in \mathcal{P}\left({ }^{k} l_{p}: l_{p}\right) \quad(1<p<\infty)$, if $v(Q)=0$, then $\|Q\|=0$.

\section{Introduction}

Given a complex or real Banach space $E$ we write $B_{E}$ for the closed unit ball and $S_{E}$ for the unit sphere of $E$. The dual space of $E$ is denoted by $E^{*}$. For $k \in \mathbb{N}$, a mapping $P: E \rightarrow E$ is called a (continuous) $k$-homogeneous polynomial if there is a $k$-multilinear (continuous) mapping $A: E \times \cdots \times E \rightarrow E$ such that $P(x)=A(x, \ldots, x)$ for every $x \in E . \mathcal{P}\left({ }^{k} E: E\right)$ denotes the Banach space of all $k$-homogeneous continuous polynomials from $E$ into itself with the norm $\|P\|=\sup _{x \in B_{E}}\|P(x)\|$. We refer to [6] for background of polynomials on a Banach space. Let

$$
\Pi(E):=\left\{\left(x, x^{*}\right): x \in S_{E}, x^{*} \in S_{E^{*}}, x^{*}(x)=1\right\} .
$$

The numerical radius of $P$ is defined [3] by

$$
v(P):=\sup \left\{\left|x^{*}(P x)\right|:\left(x, x^{*}\right) \in \Pi(E)\right\} .
$$

The polynomial numerical index of order $k$ of $E$ is defined [4] by

$$
\begin{aligned}
n^{(k)}(E) & :=\inf \left\{v(P): P \in \mathcal{P}\left({ }^{k} E: E\right),\|P\|=1\right\} \\
& =\sup \left\{M \geq 0:\|P\| \leq M v(P) \text { for all } P \in \mathcal{P}\left({ }^{k} E: E\right)\right\} .
\end{aligned}
$$

Of course, $n^{(1)}(E)$ is the classical numerical index of $E$. Note that $0 \leq n^{(k)}(E) \leq 1$, and $n^{(k)}(E)>0$ if and only if $v(\cdot)$ is a norm on $\mathcal{P}\left({ }^{k} E: E\right)$ equivalent to the usual

Received July 11, 2012; accepted August 23, 2012.

2010 Mathematics Subject Classification: 46A22, 46G20, 46G25.

Key words and phrases: Homogeneous polynomials, polynomial numerical index.

This research was supported by the Basic Science Research Program through the National

Research Foundation of Korea(NRF) funded by the Ministry of Education, Science and Technology (2010-0009854). 
norm. It is obvious that if $E_{1}, E_{2}$ are isometrically isomorphic Banach spaces, then $n^{(k)}\left(E_{1}\right)=n^{(k)}\left(E_{2}\right)$.

The concept of the classical numerical index (in our terminology, the polynomial numerical index of order 1) was first suggested by G. Lumer [12]. In [4] the authors proved $n^{(k)}(C(K))=1$ when $C(K)$ is the complex spaces and some inequality $n^{(k)}(E) \leq n^{(k-1)}(E) \leq \frac{k^{\left(k+\frac{1}{k-1}\right)}}{(k-1)^{k-1}} n^{(k)}(E)$ for every Banach space $E$. It was shown that $n^{(k)}\left(E^{* *}\right) \leq n^{(k)}(E)$. The authors [10] found a lower bound for the polynomial numerical index of real lush spaces. They used this bound to compute the polynomial numerical index of order 2 of the real spaces $c_{0}, \ell_{1}$ and $\ell_{\infty}$. In fact, they showed that for the real spaces $X=c_{0}, l_{1}, l_{\infty}, n^{(2)}(X)=1 / 2$. They also presented an example of a real Banach space $X$ whose polynomial numerical indices are positive while the ones of its bidual are zero. We refer to $([1-5,7-12])$ for some results about the polynomial numerical index. For general information and background on numerical ranges, we refer to [1-2].

In this paper, we show that for $1<p<\infty, k, m \in \mathbb{N}, n^{(k)}\left(l_{p}\right)=\inf \left\{n^{(k)}\left(l_{p}^{m}\right)\right.$ : $m \in \mathbb{N}\}$ and that for any positive measure $\mu, n^{(k)}\left(L_{p}(\mu)\right) \geq n^{(k)}\left(l_{p}\right)$. We also prove that for every $Q \in \mathcal{P}\left({ }^{k} l_{p}: l_{p}\right) \quad(1<p<\infty)$, if $v(Q)=0$, then $\|Q\|=0$.

\section{Results}

For $1<p<\infty$ and $m \in \mathbb{N}, l_{p}^{m}$ denotes $\mathbb{K}^{m}$ endowed with the usual $p$-norm, where $\mathbb{K}=\mathbb{R}$ or $\mathbb{C}$. We may consider $l_{p}^{m}$ as a subspace of $l_{p}$. Let $\left\{e_{n}\right\}_{\mathbb{N}}$ be the canonical basis of $l_{p}$ and $\left\{e_{n}^{*}\right\}_{n \in \mathbb{N}}$ the biorthogonal functionals associated to $\left\{e_{n}\right\}_{n \in \mathbb{N}}$. Note that in general if $X$ is a Banach space and $Y$ is a subspace of $X$ there is no comparison between $n^{(k)}(X)$ and $n^{(k)}(Y)$ for $k \in \mathbb{N}$.

Theorem 2.1. Let $1<p<\infty$ and $k \in \mathbb{N}$ be fixed. Then $n^{(k)}\left(l_{p}\right)=\inf \left\{n^{(k)}\left(l_{p}^{m}\right)\right.$ : $m \in \mathbb{N}\}$ and the sequence $\left\{n^{(k)}\left(l_{p}^{m}\right)\right\}_{m \in \mathbb{N}}$ is decreasing.

Proof. We proceed by steps. Let $m \in \mathbb{N}$. We define $P_{\{1, \cdots, m\}}: l_{p} \rightarrow l_{p}^{m}$ by $P_{\{1, \cdots, m\}}\left(\sum_{j=1}^{\infty} \lambda_{j} e_{j}\right)=\sum_{j=1}^{m} \lambda_{j} e_{j}$.

Step 1: The sequence $\left\{n^{(k)}\left(l_{p}^{m}\right)\right\}_{m \in \mathbb{N}}$ is decreasing.

Proof of Step 1. Let $Q \in S_{\mathcal{P}\left({ }^{k} l_{p}^{m}: l_{p}^{m}\right)}$. We define $\tilde{Q} \in \mathcal{P}\left({ }^{k} l_{p}^{m+1}: l_{p}^{m+1}\right)$ by $\tilde{Q}(x)=\left(Q \circ P_{\{1, \cdots, m\}}(x), 0\right)$ for $x \in l_{p}^{m+1}$. It is obvious that $\tilde{Q} \in S_{\mathcal{P}}\left({ }^{k} l_{p}^{m+1}: l_{p}^{m+1}\right)$.

Claim A: $v(Q)=v(\tilde{Q})$

Let $\left(x, x^{*}\right) \in \Pi\left(l_{p}^{m}\right)$. Then $\left((x, 0),\left(x^{*}, 0\right)\right) \in \Pi\left(l_{p}^{m+1}\right)$ and

(*) $\left|x^{*}(Q(x))\right|=\left|\left(x^{*}, 0\right)(\tilde{Q}((x, 0)))\right| \leq v(\tilde{Q})$.

By taking supremum in the left side of $(*)$ over $\left(x, x^{*}\right) \in \Pi\left(l_{p}^{m}\right)$, we have $v(Q) \leq$ $v(\tilde{Q})$. For the reverse inequality let $\epsilon>0$. Then there exists $z_{0}:=\sum_{j=1}^{m+1} a_{j} e_{j} \in$ 
$S_{l_{p}^{m+1}}$ such that $\left(z_{0}, \sum_{j=1}^{m+1} \operatorname{sign}\left(a_{j}\right)\left|a_{j}\right|^{p-1} e_{j}^{*}\right) \in \Pi\left(l_{p}^{m+1}\right)$ and

$$
\begin{aligned}
v(\tilde{Q})-\epsilon< & \left.\left|\sum_{j=1}^{m+1} \operatorname{sign}\left(a_{j}\right)\right| a_{j}\right|^{p-1} e_{j}^{*}\left(\tilde{Q}\left(z_{0}\right)\right) \mid \\
= & \left.\left|\sum_{j=1}^{m} \operatorname{sign}\left(a_{j}\right)\right| a_{j}\right|^{p-1} e_{j}^{*}\left(Q\left(\sum_{j=1}^{m} a_{j} e_{j}\right)\right) \mid \\
= & \left.C^{k+p-1}\left|\sum_{j=1}^{m} \operatorname{sign}\left(a_{j}\right)\right| \frac{a_{j}}{C}\right|^{p-1} e_{j}^{*}\left(Q\left(\sum_{j=1}^{m} \frac{a_{j}}{C} e_{j}\right)\right) \mid \\
& \left(\text { where } C:=\left(\sum_{j=1}^{m}\left|a_{j}\right|^{p}\right)^{\frac{1}{p}} \leq 1\right) \\
\leq & \left.\left|\sum_{j=1}^{m} \operatorname{sign}\left(a_{j}\right)\right| \frac{a_{j}}{C}\right|^{p-1} e_{j}^{*}\left(Q\left(\sum_{j=1}^{m} \frac{a_{j}}{C} e_{j}\right)\right) \mid \\
\leq & v(Q), \text { because }\left(\sum_{j=1}^{m} \frac{a_{j}}{C} e_{j}, \sum_{j=1}^{m} \operatorname{sign}\left(a_{j}\right)\left|\frac{a_{j}}{C}\right|^{p-1} e_{j}^{*}\right) \in \Pi\left(l_{p}^{m}\right),
\end{aligned}
$$

which show $v(\tilde{Q}) \leq v(Q)$. Thus $v(Q)=v(\tilde{Q})$.

It follows that

$$
\begin{aligned}
n^{(k)}\left(l_{p}^{m}\right) & =\inf _{Q \in S_{\mathcal{P}\left(k_{p} l_{p}^{m}: l_{p}^{m}\right)}} v(Q) \\
& =\inf _{Q \in S_{\mathcal{P}\left(k_{p} l_{p}^{m}: l_{p}^{m}\right)}^{m}} v(\tilde{Q}) \\
& \geq \inf _{R \in S_{\mathcal{P}\left(l_{p}^{m} l_{1}^{m}: l_{p}^{m+1}\right)}} v(R) \\
& =n^{(k)}\left(l_{p}^{m+1}\right) .
\end{aligned}
$$

Step 2: $n^{(k)}\left(l_{p}\right) \leq n^{(k)}\left(l_{p}^{m}\right)$ for every $m \in \mathbb{N}$

Proof of Step 2. Let $Q \in S_{\mathcal{P}\left({ }^{k} l_{p}^{m}: l_{p}^{m}\right)}$. We define $\tilde{Q} \in \mathcal{P}\left({ }^{k} l_{p}: l_{p}\right)$ by $\tilde{Q}(z)=$ $\left(Q \circ P_{\{1, \cdots, m\}}(z), 0,0, \cdots\right)$ for $z \in l_{p}$. It is obvious that $\tilde{Q} \in S_{\mathcal{P}\left({ }^{k} l_{p}: l_{p}\right)}$. By the same argument as in Step 1, we have $v(\tilde{Q}) \leq v(Q)$. Thus it follows.

Step 3: $\lim _{m \rightarrow \infty} n^{(k)}\left(l_{p}^{m}\right)=n^{(k)}\left(l_{p}\right)$

Proof of Step 3. Let $Q \in S_{\mathcal{P}\left(k l_{p}: l_{p}\right)}$. For each $m \in \mathbb{N}$, we define $Q_{m} \in \mathcal{P}\left({ }^{k} l_{p}^{m}\right.$ : $\left.l_{p}^{m}\right)$ by $Q_{m}(x)=P_{\{1, \cdots, m\}} \circ Q(x, 0,0, \cdots)$ for $x \in l_{p}^{m}$. It is obvious that $\left\|Q_{m}\right\| \leq$ $1,\left\|Q_{m}\right\| \leq\left\|Q_{m+1}\right\|$ and $v\left(Q_{m}\right) \leq v(Q)$. For each $m \in \mathbb{N}$, we define $\tilde{Q}_{m} \in \mathcal{P}\left({ }^{k} l_{p}: l_{p}\right)$ by $\tilde{Q}_{m}(z)=\left(Q_{m} \circ P_{\{1, \cdots, m\}}(z), 0,0, \cdots\right)$ for $z \in l_{p}$. By the argument in Step 1 , $v\left(\tilde{Q}_{m}\right)=v\left(Q_{m}\right)$.

Claim B: $\lim _{m \rightarrow \infty}\left\|Q_{m}\right\|=1$ 
Let $\epsilon>0$. Choose $x_{0} \in S_{l_{p}}$ such that $\left\|Q\left(x_{0}\right)\right\|>1-\epsilon$. By continuity of $Q$ at $x_{0}$ it follows that

$$
\begin{aligned}
& \left\|Q_{m} \circ P_{\{1, \cdots, m\}}\left(x_{0}\right)-Q\left(x_{0}\right)\right\| \\
= & \left\|P_{\{1, \cdots, m\}} \circ Q \circ P_{\{1, \cdots, m\}}\left(x_{0}\right)-P_{\{1, \cdots, m\}} \circ Q\left(x_{0}\right)\right\|+\left\|P_{\{1, \cdots, m\}} \circ Q\left(x_{0}\right)-Q\left(x_{0}\right)\right\| \\
\leq & \left\|Q \circ P_{\{1, \cdots, m\}}\left(x_{0}\right)-Q\left(x_{0}\right)\right\|+\left\|P_{\{1, \cdots, m\}} \circ Q\left(x_{0}\right)-Q\left(x_{0}\right)\right\| \rightarrow 0 \text { as } m \rightarrow \infty .
\end{aligned}
$$

Choose $N_{0} \in \mathbb{N}$ such that $\left\|Q_{m} \circ P_{\{1, \cdots, m\}}\left(x_{0}\right)-Q\left(x_{0}\right)\right\|<\epsilon$ for all $m \geq N_{0}$. Then for all $m \geq N_{0}, 1 \geq\left\|Q_{m}\right\| \geq\left\|Q_{m} \circ P_{\{1, \cdots, m\}}\left(x_{0}\right)\right\|>1-2 \epsilon$, which shows Claim B.

Claim C: $\lim _{m \rightarrow \infty} v\left(Q_{m}\right)=v(Q)$

There exists $\left(y_{0}, y^{*}\right) \in \Pi\left(l_{p}\right)$ such that $\left|y^{*}\left(Q\left(y_{0}\right)\right)\right|>v(Q)-\epsilon$. Let $y_{0}:=$ $\sum_{j=1}^{\infty} b_{j} e_{j}$. Then $y^{*}=\sum_{j=1}^{\infty} \operatorname{sign}\left(b_{j}\right)\left|b_{j}\right|^{p-1} e_{j}^{*}$. For $m \in \mathbb{N}$, we define $y_{0}^{(m)}:=\sum_{j=1}^{m-1} b_{j} e_{j}+\left(\sum_{j=m}^{\infty}\left|b_{j}\right|^{p}\right)^{\frac{1}{p}} e_{m}$ and $y_{m}^{*}:=\sum_{j=1}^{m-1} \operatorname{sign}\left(b_{j}\right)\left|b_{j}\right|^{p-1} e_{j}^{*}+$ $\left(\sum_{j=m}^{\infty}\left|b_{j}\right|^{p}\right)^{\frac{p-1}{p}} e_{m}^{*}$. It is obvious that $\left(y_{0}^{(m)}, y_{m}^{*}\right) \in \Pi\left(l_{p}\right)$ and $\lim _{m \rightarrow \infty}\left\|y_{0}-y_{0}^{(m)}\right\|=$ $0=\lim _{m \rightarrow \infty}\left\|y^{*}-y_{m}^{*}\right\|$. Note that $\lim _{m \rightarrow \infty} y_{m}^{*}\left(Q\left(y_{0}^{(m)}\right)\right)=y^{*}\left(Q\left(y_{0}\right)\right)$. Indeed,

$$
\begin{aligned}
& \left|y_{m}^{*}\left(Q\left(y_{0}^{(m)}\right)\right)-y^{*}\left(Q\left(y_{0}\right)\right)\right| \\
\leq & \left|y_{m}^{*}\left(Q\left(y_{0}^{(m)}\right)\right)-y^{*}\left(Q\left(y_{0}^{(m)}\right)\right)\right|+\left|y^{*}\left(Q\left(y_{0}^{(m)}\right)\right)-y^{*}\left(Q\left(y_{0}\right)\right)\right| \\
\leq & \left\|y_{m}^{*}-y^{*}\right\|\left\|Q\left(y_{0}^{(m)}\right)\right\|+\left\|Q\left(y_{0}^{(m)}\right)-Q\left(y_{0}\right)\right\| \\
\leq & \left\|y_{m}^{*}-y^{*}\right\|+\left\|Q\left(y_{0}^{(m)}\right)-Q\left(y_{0}\right)\right\| \rightarrow 0 \text { as } m \rightarrow \infty .
\end{aligned}
$$

Choose $N_{1} \in \mathbb{N}$ such that $\left|y_{m}^{*}\left(Q\left(y_{0}^{(m)}\right)\right)\right|>v(Q)-\epsilon$ for all $m \geq N_{1}$. It is easy to show that for all $m \geq N_{1}, y_{N_{1}}^{*}\left(\tilde{Q}_{m}\left(y_{0}^{\left(N_{1}\right)}\right)\right)=y_{N_{1}}^{*}\left(Q\left(y_{0}^{\left(N_{1}\right)}\right)\right)$. It follows that for all $m \geq N_{1}$,

$$
\begin{aligned}
v(Q)-\epsilon & <\left|y_{N_{1}}^{*}\left(Q\left(y_{0}^{\left(N_{1}\right)}\right)\right)\right| \\
& =\left|y_{N_{1}}^{*}\left(\tilde{Q}_{m}\left(y_{0}^{\left(N_{1}\right)}\right)\right)\right| \\
& \leq v\left(\tilde{Q}_{m}\right)=v\left(Q_{m}\right) \\
& \leq v(Q),
\end{aligned}
$$

which show $\lim _{m \rightarrow \infty} v\left(Q_{m}\right)=v(Q)$. Thus we have

$$
\begin{aligned}
& (* *) v(Q)=\lim _{m \rightarrow \infty} v\left(Q_{m}\right) \\
= & \limsup _{m \rightarrow \infty}\left[v\left(\frac{Q_{m}}{\left\|Q_{m}\right\|}\right)\left\|Q_{m}\right\|\right] \\
= & \limsup _{m \rightarrow \infty} v\left(\frac{Q_{m}}{\left\|Q_{m}\right\|}\right) \lim _{m \rightarrow \infty}\left\|Q_{m}\right\| \\
= & \limsup _{m \rightarrow \infty} v\left(\frac{Q_{m}}{\left\|Q_{m}\right\|}\right) \quad \text { (by claim B) } \\
\geq & \limsup _{m \rightarrow \infty} n^{(k)}\left(l_{p}^{m}\right)
\end{aligned}
$$


Taking the infimum in the left side of $(* *)$ over $Q \in S_{\mathcal{P}\left({ }^{k} l_{p}: l_{p}\right)}$, we have $n^{(k)}\left(l_{p}\right) \geq$ $\limsup _{m \rightarrow \infty} n^{(k)}\left(l_{p}^{m}\right)$. By Step 2, we have $n^{(k)}\left(l_{p}\right) \leq \liminf _{m \rightarrow \infty} n^{(k)}\left(l_{p}^{m}\right)$. Thus $\lim _{m \rightarrow \infty} n^{(k)}\left(l_{p}^{m}\right)=n^{(k)}\left(l_{p}\right)$. Therefore, we complete the proof.

Theorem 2.2. Let $1<p<\infty$. Let $Q \in \mathcal{P}\left({ }^{k} l_{p}: l_{p}\right)$. Then $v(Q)=0$ if and only if $\|Q\|=0$.

Proof. It is enough to show that if $v(Q)=0$, then $Q=0$. We will show that $Q_{m}:=\left.P_{\{1, \cdots, m\}} \circ Q\right|_{\operatorname{span}\left\{e_{1}, \cdots, e_{m}\right\}}: l_{p}^{m} \rightarrow l_{p}^{m}$ is the zero polynomial for every $m \in \mathbb{N}$. Write

$$
Q_{m}\left(\sum_{k=1}^{m} x_{k} e_{k}\right)=\sum_{k_{1}+\cdots+k_{m}=m, 0 \leq k_{1}, \cdots, k_{m} \leq m} \frac{m !}{k_{1} ! \cdots k_{m} !} x_{1}^{k_{1}} x_{2}^{k_{2}} \cdots x_{m}^{k_{m}} A_{m}\left(e_{k_{1}}, \cdots, e_{k_{m}}\right),
$$

where $A_{m}$ is the corresponding symmetric $k$-linear mapping to the $k$-homogeneous polynomial $Q_{m}$. Let $a_{k_{1} \cdots k_{m}}:=A_{m}\left(e_{k_{1}}, \cdots, e_{k_{m}}\right) \in l_{p}^{m}$.

Let $p_{1}:=0$ and $p_{n}$ be the $n$-th prime $(n \geq 2)$. Let $0 \leq t \leq 1$ be fixed and $q \in \mathbb{R}$ with $1 / p+1 / q=1$. Put

$$
y:=\frac{t^{\sqrt{p_{1}}} e_{1}+t^{\sqrt{p_{2}}} e_{2}+\cdots+t^{\sqrt{p_{m}}} e_{m}}{\left(1+t^{p \sqrt{p_{2}}}+\cdots+t^{p \sqrt{p_{m}}}\right)^{1 / p}}
$$

and

$$
y^{*}:=\frac{t^{(p-1) \sqrt{p_{1}}} e_{1}^{*}+t^{(p-1) \sqrt{p_{2}}} e_{2}^{*}+\cdots+t^{(p-1) \sqrt{p_{m}}} e_{m}^{*}}{\left(1+t^{p \sqrt{p_{2}}}+\cdots+t^{p \sqrt{p_{m}}}\right)^{1 / q}} .
$$

Then $\left(y, y^{*}\right) \in \Pi\left(l_{p}^{m}\right)$.

Claim: $a_{k_{1} \cdots k_{m}}=0$ for every $k_{1}, \cdots, k_{m}$

It follows that for every $0 \leq t \leq 1$,

$$
\begin{aligned}
0= & y^{*}\left(Q_{m}(y)\right) \\
= & \frac{1}{\left(1+t^{p \sqrt{p_{2}}}+\cdots+t^{p \sqrt{p_{m}}}\right)^{1 / q+k / p}} \times \\
& \left(t^{(p-1) \sqrt{p_{1}}} e_{1}^{*}+t^{(p-1) \sqrt{p_{2}}} e_{2}^{*}+\cdots+t^{(p-1) \sqrt{p_{m}}} e_{m}^{*}\right) \\
& \left(Q_{m}\left(t^{\sqrt{p_{1}}} e_{1}+t^{\sqrt{p_{2}}} e_{2}+\cdots+t^{\sqrt{p_{m}}} e_{m}\right)\right),
\end{aligned}
$$

so

$$
\begin{aligned}
0 & =\left(t^{(p-1) \sqrt{p_{1}}} e_{1}^{*}+t^{(p-1) \sqrt{p_{2}}} e_{2}^{*}+\cdots+t^{(p-1) \sqrt{p_{m}}} e_{m}^{*}\right) \\
& \left(Q_{m}\left(t^{\sqrt{p_{1}}} e_{1}+t^{\sqrt{p_{2}}} e_{2}+\cdots+t^{\sqrt{p_{m}}} e_{m}\right)\right) \\
& \sum_{k_{1}+\cdots+k_{m}=m, 0 \leq k_{1}, \cdots, k_{m} \leq m} t^{\sqrt{p_{k_{2}}}+\cdots+\sqrt{p_{k_{m}}}} \frac{m !}{k_{1} ! \cdots k_{m} !} e_{1}^{*}\left(a_{k_{1} \cdots k_{m}}\right) \\
+ & \sum_{2 \leq j \leq m}\left[\sum_{k_{1}+\cdots+k_{m}=m, 0 \leq k_{1}, \cdots, k_{m} \leq m} \sum^{\sqrt{p_{k_{2}}}+\cdots+\sqrt{p_{k_{m}}}+(p-1) \sqrt{p_{k_{j}}}} \frac{m !}{k_{1} ! \cdots k_{m} !} e_{j}^{*}\left(a_{k_{1} \cdots k_{m}}\right)\right] .
\end{aligned}
$$


Note that the elements of the set

$$
\begin{gathered}
\left\{\sqrt{p_{k_{2}}}+\cdots+\sqrt{p_{k_{m}}}, \sqrt{p_{k_{2}}}+\cdots+\sqrt{p_{k_{m}}}+(p-1) \sqrt{p_{k_{j}}}:\right. \\
\left.k_{1}+\cdots+k_{m}=m, 0 \leq k_{1}, \cdots, k_{m} \leq m, 2 \leq j \leq m\right\}
\end{gathered}
$$

are different. Thus $e_{j}^{*}\left(a_{k_{1} \cdots k_{m}}\right)=0$ for every $1 \leq j \leq m$, which show $a_{k_{1} \cdots k_{m}}=0$ for every $k_{1}, \cdots, k_{m}$. Therefore, $Q_{m}=0$. Let $x=\sum_{k=1}^{\infty} x_{k} e_{k} \in l_{p}$ be fixed. By continuity of $Q$ at $x$, we have

$$
Q(x)=\lim _{m \rightarrow \infty} Q_{m}(x)=0 .
$$

Corollary 2.3. Let $1<p<\infty$. Then for every $k, m \in \mathbb{N}$, we have $n^{(k)}\left(l_{p}^{m}\right)>0$.

Proof. Assume that $n^{(k)}\left(l_{p}^{m}\right)=0$ for some $k, m \in \mathbb{N}$. Since the unit sphere of the finite dimensional space $\mathcal{P}\left({ }^{k} l_{p}^{m}: l_{p}^{m}\right)$ is compact, there exists some $Q \in \mathcal{P}\left({ }^{k} l_{p}^{m}: l_{p}^{m}\right)$ such that $\|Q\|=1$ and $v(Q)=0$. Theorem 2.2 shows that $Q=0$, which is impossible.

Let $(\Omega, \Sigma)$ be a measurable space and $\mu$ a positive measure on $\Omega$. We denote by $\mathcal{P}$ the collection of all partitions $\pi$ of $\Omega$ into finitely many pairwise disjoint members of $\Sigma$ with finite strictly positive measures. We order this collection by $\pi_{1} \leq \pi_{2}$ whenever each member of $\pi_{1}$ is the union of members of $\pi_{2}$. So $\mathcal{P}$ is a directed set. For each $\pi=\left\{E_{1}, \cdots, E_{m}\right\} \in \mathcal{P}$, we associate the subspace $V_{\pi}$ of $L_{p}(\mu)$ defined by $V_{\pi}=\left\{\sum_{i=1}^{m} a_{i} 1_{E_{i}}: a_{i} \in \mathbb{K}\right\}$. By $P_{\pi}$ we denote the projection of $L_{p}(\mu)$ onto $V_{\pi}$ defined by

$$
P_{\pi}(f)=\sum_{i=1}^{m}\left[\frac{1}{\mu\left(E_{i}\right)} \int_{E_{i}} f(t) d t\right] 1_{E_{i}}
$$

for all $f \in L_{p}(\mu) . V$ denotes the union of all subspaces $V_{\pi}$ of $L_{p}(\mu)$. We recall that $V$ is a dense subspace of $L_{p}(\mu)$, thus, for each $f \in L_{p}(\mu)$, the sequence $\left\{P_{\pi}(f)\right\}_{\pi}$ converges to $f$ in $L_{p}(\mu)$.

We recall the following well-known result.

Theorem 2.4 For $1<p<\infty$ and for every partition $\pi=\left\{E_{1}, \cdots, E_{m}\right\} \in \mathcal{P}$, the subspace $V_{\pi}$ is isometrically isomorphic to $l_{p}^{m}$. Thus $n^{(k)}\left(V_{\pi}\right)=n^{(k)}\left(l_{p}^{m}\right)$ for every $k \in \mathbb{N}$.

Theorem 2.5. Let $1<p<\infty$ and $k \in \mathbb{N}$. Then for any positive measure $\mu$,

$$
n^{(k)}\left(L_{p}(\mu)\right) \geq n^{(k)}\left(l_{p}\right) .
$$

Proof. Let $Q \in S_{\mathcal{P}\left({ }^{k} L_{p}(\mu): L_{p}(\mu)\right)}$. Let $\epsilon>0$. Choose $x_{0} \in S_{L_{p}(\mu)}$ such that $\left\|Q\left(x_{0}\right)\right\|>1-\epsilon$. By uniform continuity of $Q$ on the closed unit ball of $L_{p}(\mu)$, there exists some $\delta>0$ such that $x, y \in B_{L_{p}(\mu)}$ with $\|x-y\|<\delta$ implies that $\|Q(x)-Q(y)\|<\epsilon$. Choose $\pi_{0} \in \mathcal{P}$ such that $\left\|x_{0}-P_{\pi_{0}}\left(x_{0}\right)\right\|<\delta$. Since $\left\|P_{\pi_{0}}\left(x_{0}\right)\right\| \leq 1$, we have $\left\|Q\left(x_{0}\right)-Q \circ P_{\pi_{0}}\left(x_{0}\right)\right\|<\epsilon$. Thus $\left\|Q \circ P_{\pi_{0}}\left(x_{0}\right)\right\|>$ $\left\|Q\left(x_{0}\right)\right\|-\epsilon>1-2 \epsilon$. Thus we can choose $\pi_{1}=\left\{E_{1}, \cdots, E_{m}\right\} \in \mathcal{P}$ such that 
$\pi_{1} \geq \pi_{0}$ and $\left\|P_{\pi_{1}} \circ Q \circ P_{\pi_{0}}\left(x_{0}\right)\right\|>1-2 \epsilon$. We define $R \in \mathcal{P}\left({ }^{k} V_{\pi_{1}}: V_{\pi_{1}}\right)$ by $R\left(P_{\pi_{1}}(x)\right)=P_{\pi_{1}} \circ Q \circ P_{\pi_{1}}(x)$ for $x \in L_{p}(\mu)$. Obviously $\|R\| \leq 1$. It follows that

$$
\begin{aligned}
(\sharp)\|R\| & \geq\left\|R\left(\frac{P_{\pi_{0}}\left(x_{0}\right)}{\left\|P_{\pi_{0}}\left(x_{0}\right)\right\|}\right)\right\|=\frac{\left\|R\left(P_{\pi_{0}}\left(x_{0}\right)\right)\right\|}{\left\|P_{\pi_{0}}\left(x_{0}\right)\right\|^{k}} \\
& \geq\left\|R\left(P_{\pi_{0}}\left(x_{0}\right)\right)\right\| \\
& \geq\left\|P_{\pi_{1}} \circ Q \circ P_{\pi_{0}}\left(x_{0}\right)\right\| \\
& >1-2 \epsilon .
\end{aligned}
$$

Thus

$$
\begin{aligned}
(\sharp \sharp) v(R) & \geq n^{(k)}\left(V_{\pi_{1}}\right)\|R\| \\
& >n^{(k)}\left(V_{\pi_{1}}\right)(1-2 \epsilon)(\text { by } \sharp) \\
& =n^{(k)}\left(l_{p}^{m}\right)(1-2 \epsilon)(\text { by Theorem 2.4) } \\
& \geq n^{(k)}\left(l_{p}\right)(1-2 \epsilon) \text { (by Theorem 2.1). }
\end{aligned}
$$

Since $V_{\pi_{1}}$ is a finite dimensional space, there exists $\left(y_{0}, y^{*}\right) \in \Pi\left(V_{\pi_{1}}\right)$ such that $v(R)=\left|y^{*}\left(R\left(y_{0}\right)\right)\right|$. It follows that

$$
\begin{aligned}
v(R) & =\left|y^{*}\left(R\left(y_{0}\right)\right)\right|=\left|y^{*}\left(P_{\pi_{1}} \circ Q\left(y_{0}\right)\right)\right| \\
& =\left|P_{\pi_{1}}^{*} \circ y^{*}\left(Q\left(y_{0}\right)\right)\right| \\
& \leq v(Q), \text { because }\left(y_{0}, P_{\pi_{1}}^{*} \circ y^{*}\right) \in \Pi\left(V_{\pi_{1}}\right) .
\end{aligned}
$$

By $(\sharp \sharp)$, we have $(\sharp \sharp) \quad v(Q) \geq v(R)>n^{(k)}\left(l_{p}\right)(1-2 \epsilon)$. By taking infimum in the left side of $(\sharp \sharp)$ over $Q \in S_{\mathcal{P}\left({ }^{k} L_{p}(\mu): L_{p}(\mu)\right)}$, we conclude the proof.

Acknowledgements The author would like to thank the referee for his comments and suggestions in improving this paper in both substance and presentation.

\section{References}

[1] F. F. Bonsall and J. Duncan, Numerical Ranges of Operators on Normed Spaces and of Elements of Normed Algebras, London Math. Soc. Lecture Note Ser. 2, Cambridge Univ. Press, 1971.

[2] F. F. Bonsall and J. Duncan, Numerical Ranges II, London Math. Soc. Lecture Note Ser. 10, Cambridge Univ. Press, 1973.

[3] Y. S. Choi and S. G. Kim, Norm or numerical radius attaining multilinear mappings and polynomials, J. London Math. Soc., 54 (1996), 135-147.

[4] Y. S. Choi, D. Garcia, S. G. Kim and M. Maestre, The polynomial numerical index of a Banach space, Proc. Edinburgh Math. Soc., 49 (2006), 39-52. 
[5] Y. S. Choi, D. Garcia, S. G. Kim and M. Maestre, Composition, numerical range and Aron-Berner extension, Math. Scand., 103 (2008), 97-110.

[6] S. Dineen, Complex Analysis on Infinite Dimensional Spaces, Springer-Verlag, London, 1999.

[7] J. Duncan, C. M. McGregor, J. D. Pryce and A. J. White, The numerical index of a normed space, J. London Math. Soc., 2 (1970), 481-488.

[8] S. G. Kim, Norm and numerical radius of 2-homogeneous polynomials on the real space $l_{p}^{2},(1<p<\infty)$, Kyungpook Math. J., 48 (2008), 387-393.

[9] S. G. Kim, Three kinds of numerical indices of a Banach space, Math. Proc. Royal Irish Acad., 112A (2012), 21-35.

[10] S. G. Kim, M. Martin and J. Meri, On the polynomial numerical index of the real spaces $c_{0}, \ell_{1}, \ell_{\infty}$, J. Math. Anal. Appl., 337 (2008), 98-106.

[11] H. J. Lee, Banach spaces with polynomial numerical index 1, Bull. Lond. Math. Soc., 40 (2008), 193-198.

[12] G. Lumer, Semi-inner-product spaces, Trans. Amer. Math. Soc., 100 (1961), 29-43. 